\title{
Slow-light induced interference with stacked optical precursors for square input pulses
}

\author{
Heejeong Jeong ${ }^{1,3}$ and Shengwang $\mathrm{Du}^{2,4}$ \\ ${ }^{1}$ Samsung Electronics Co., Ltd. Suwon, Gyeonggi 443-742, Korea \\ ${ }^{2}$ Department of Physics, The Hong Kong University of Science and Technology, \\ Clear Water Bay, Kowloon, Hong Kong, China \\ 3 jhj413@gmail.com \\ ${ }^{4}$ dusw@ust.hk
}

Compiled December 29, 2009

We theoretically study the stacked optical transients generated from a series of square pulses passing through a cold atomic ensemble. Using the hybrid analysis and fast Fourier transform, we identify the stacked coherent transients (Segard et al. 1987) as optical precursors. With slow light and electromagnetically induced transparency, we obtain nearly $700 \%$ transmitted intensity at the transient spikes resulting from the interference between the delayed main field and the stacked optical precursors. (c) 2009 Optical Society of America OCIS codes: $070.7345,270.1670$.

Introduction - Optical precursors [1,2] in anomalously dispersive media, analogous to coherent transients [3,4], recently became of great interest due to their nondecaying properties [5] as well as their instantaneous speed of light in vacuum $c$. The concept was first introduced by Varoquaux et. al. [6] by using "time-domain analysis". However, the analogy was not accepted by both communities of optical precursors [2] and coherent transients $[3,4]$ because the conventional concept of optical precursors was established based on strongly dispersive far-off resonance condition $[1,2]$.

Recent experimental study of optical precursors in cold atoms [5] has triggered unifying both concepts $[7,8]$ to include phenomena such as resonantly filtered gammaray [9] and small area $(0 \pi)$ pulses [10]. Motivated by the observation of precursory spikes at the leading edge of biphoton correlation with electromagnetically induced transparency (EIT) $[11,12]$, the classical step-on pulse transmission in EIT medium has been studied theoretically $[13,14]$. Most recently, observation of optical precursors at both rising and falling edges of a square pulse passing through an EIT system has been reported [15]. Combining EIT and optical precursors makes it possible to control the delay between precursors and main signal for the application of active optical devices.

In coherent transients, one of the interesting effects is the "stacking of the coherent transients" by Macke et. al. [4]. About 300\% output with respect to the input intensity was achieved by sending a series of square pulses through an optically thick single Lorentz-line medium. Stacking of coherent transients at edges results in output peaks larger than the input intensity [4]. In their early works $[4,16]$, however, the possibility of optical precursors had been excluded until recently [14].

In this Letter, we first identify, in a two-level system, Macke's coherent stacks [4] as the stack of SommerfeldBrillouin precursors at the rising- and falling-edge of square pulses using the asymptotic saddle-point method.

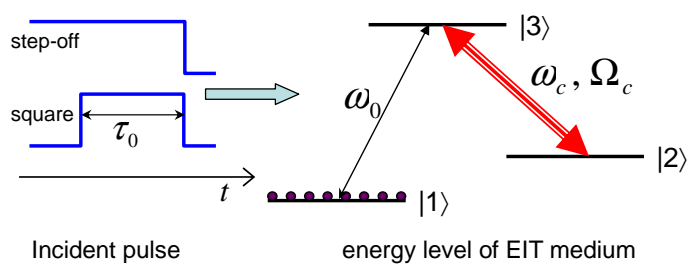

Fig. 1. (Color online) Illustration of a step-off or square pulse propagation through an EIT medium. The carrier frequency of the incident pulse $\omega_{p}=\omega_{0}$ is on resonance of the transition $|1\rangle \rightarrow|3\rangle$. The coupling laser $\left(\omega_{c}\right)$ is on resonance of the transition $|2\rangle \rightarrow|3\rangle$ with a Rabi frequency of $\Omega_{c}$.

In an optically thick EIT medium, we take the hybrid method to describe the relevant main signal and precursors [13]. The hybrid method for a Heaviside step-on modulated incident pulse $\Theta(t)$ is modified to describe a step-off modulated pulse $\Theta(-t)$. The result shows how delayed main signal (slow light) interferes with the transients (precursors) when the steady external laser beam is suddenly turned off. The transient peak intensity turns out to be $180 \%$ with respect to the input intensity. The schematic illustration of pulse propagation is shown in Fig. 1, where the on-resonance coupling laser between the transition $|2\rangle \leftrightarrow|3\rangle$ renders a narrow transparency window on the pulse carrier frequency $\omega_{p}$ that is on resonance of the transition $|1\rangle \rightarrow|3\rangle, \omega_{0}$. Finally, we extend the single square pulse to multi-squares pulses and obtain $700 \%$ output peak transmission in the EIT medium.

Step-off pulse: $\Theta(-t)$ - The linear susceptibility of the EIT medium is given in Eq. (1) of Ref. [13] as

$$
\chi(\omega)=\frac{c \alpha_{0}}{\omega_{0}} \frac{4\left(\Delta+i \delta_{12}\right) \delta}{\Omega_{c}^{2}-4\left(\Delta+i \delta_{12}\right)(\Delta+i \delta)},
$$

where $\Delta=\omega-\omega_{0}$ is the probe laser angular frequency detuning, $\alpha_{0} z=62$ is the optical depth, $\delta=2 \pi \times 3$ 

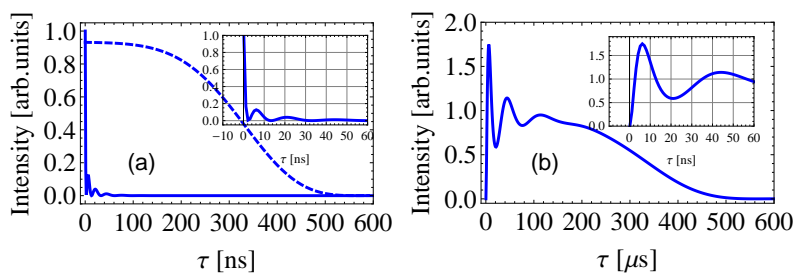

Fig. 2. (Color online) Normalized intensities of (a) total precursors (solid line) and the main signal (dashed line), and (b) total intensity at the falling edge of a step-off input pulse.

$\mathrm{MHz}$ is half of the natural linewidth, $\delta_{12}=0.005 \delta$ is the dephasing rate between the two ground levels, and $\Omega_{c}=4.20 \delta$ is the coupling laser Rabi frequency. The integral form of the transmitted pulse is given in Eq. (2) of Ref. [13]. The initial weak probe pulse is given as $E_{ \pm}(0, t)=E_{0} \Theta( \pm t) e^{-i \omega_{p} t}$ where the sign "+" stands for the step-on pulse with a rising edge and ""for the step-off pulse. Their frequency spectrums is $E_{ \pm}(0, \Delta) / E_{0}= \pm i / \Delta+\pi \delta(\Delta)$. This allows us directly apply the hybrid-asymptotic analysis in Ref. [13] to obtain the precursors for the step-off pulse by multiplying a factor of -1 to that for the step-on pulse. Thus the total Sommerfeld-Brillouin precursor for the step pulse can be obtained from Eqs.(5)-(6) of Ref. [13], and approximated as a Bessel function $[15,17]$ explaining oscillatory patterns in insets of Fig. 2 and Fig. 3.

$E_{S B \pm}(z, t) \simeq \pm E_{0} J_{0}\left(\sqrt{2 \alpha_{0} z \delta \tau}\right) \Theta(\tau) e^{-\delta \tau} e^{i\left(k_{0} z-\omega_{0} \tau\right)}$,

where $\tau=t-z / c$ and $k_{0}=\omega_{0} / c$.

To avoid the singularity of $E_{-}(0, \Delta)$ at $\Delta=0$, we express the main field $E_{M-}(z, t)$ for the step-off pulse as a convolution of the input pulse and the Green function $G_{E I T}(z, t)$ of the EIT window $\left[\omega_{0}-\Delta_{e}, \omega_{0}+\Delta_{e}\right][13]$ :

$$
E_{M-}(z, t)=E_{0} \int_{-\infty}^{0} G_{E I T}(z, t-\tau) \mathrm{d} \tau,
$$

where $\Delta_{e} \leq \Omega_{c} / 2$ is the full width of the EIT window. With EIT slow light effect, the main field is delayed and $E_{M_{-}}(z, t)$ can be approximated as a constant field within the time when the precursors occur [15].

The normalized intensities of total precursors $\left|E_{S B-}(z, t)\right|^{2} / E_{0}^{2}$ and the main signal $\left|E_{M-}(z, t)\right|^{2} / E_{0}^{2}$ are shown in Fig. 2(a). Figure 2(b) shows the total intensity $\left|E_{-}(z, t)\right|^{2} / E_{0}^{2}=\left|E_{M-}(z, t)+E_{S B_{-}}(z, t)\right|^{2} / E_{0}^{2}$. The falling edge of the main field is delayed by about $300 \mathrm{~ns}$ due to the EIT slow light effect. The interference between the main and precursor fields leads to damped oscillation with a normalized peak intensity of $180 \%$ and a decay time of $1 / \delta=52 \mathrm{~ns}$.

Square pulse: $\Theta(t)-\Theta\left(t-\tau_{0}\right)$ - Transmission of a square input pulse $E_{s q}(0, t)=E_{0}\left[\Theta(t)-\Theta\left(t-\tau_{0}\right)\right] e^{-i \omega_{p} t}$, is given by $E_{s q}(z, t)=E_{S B_{s q}}(z, t)+E_{M_{s q}}(z, t)$, where $E_{S B_{s q}}(z, t)=E_{S B+}(z, t)-E_{S B+}\left(z, t-\tau_{0}\right)$ and

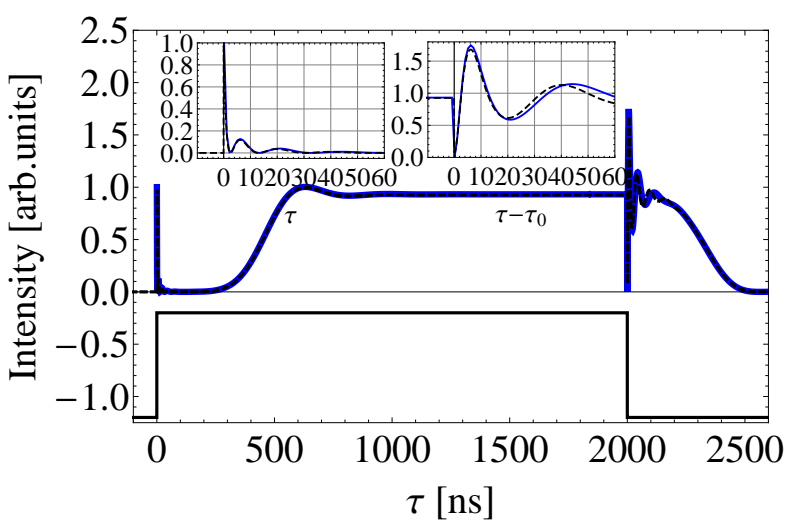

Fig. 3. (Color online) Normalized total intensities composed of optical precursors and delayed main signal in an EIT medium. Input square pulse $E(0, t)$ is given as black solid line (shifted by -1.2). Hybrid analysis [Eqs. (2)-(3), blue solid lines] is compared with FFT (black dashed lines).

$E_{M_{s q}}(z, t)=E_{M+}(z, t)-E_{M+}\left(z, t-\tau_{0}\right)$. Figure 3 shows the normalized total transmission intensity compared with fast Fourier transform (FFT) simulations. The oscillatory pattern at the falling edge is identical to the Bessel function oscillatory pattern appeared in step-off pulse [Fig. 2 (b)]. The slow-light induced interference at the falling edge is identical to the case for the step-off input pulse.

A series of square pulses: - By using Eq. (2)-(3), we first evaluate the maximum value of the stacked output for the input pulse $E(0, t) / E_{0}=\Theta(t)+\Theta\left(t-\tau_{0}\right)+$ $\sum_{i=1}^{i=5}(-1)^{i} \Theta\left(t-\tau_{0}+t_{i}\right)$, where $t_{i}=j_{1, i}^{2} /\left(2 \alpha_{0} z \delta\right)$ and $j_{1, i}$ is the $i$-th zero of $J_{1}(x)$ by considering the oscillatory pattern of precursors as originally discussed in Ref. [4].

$$
\begin{aligned}
E_{S B_{\text {stack }}}(z, t) & =E_{S B+}(z, t)+E_{S B+}\left(z, t-\tau_{0}\right) \\
& +\sum_{i=1}^{i=5}(-1)^{i} E_{S B+}\left(z, t-\tau_{0}+t_{i}\right), \quad(4) \\
E_{M_{\text {stack }}}(z, t) & =E_{0} \sum_{k=1}^{2} \int_{\tau_{0}-t_{2 k-1}}^{\tau_{0}-t_{2 k}} G_{E I T}(z, t-\tau) \mathrm{d} \tau \\
& +E_{0}\left(\int_{0}^{\tau_{0}-t_{5}}+\int_{\tau_{0}}^{\infty}\right) G_{E I T}(z, t-\tau) \mathrm{d} \tau,(5) \\
E_{\text {stack }}(z, t) & =E_{S B_{\text {stack }}}(z, t)+E_{M_{\text {stack }}}(z, t) . \quad(6)
\end{aligned}
$$

We note that, in the EIT medium, the maximum intensity from stacked coherence occurs at the step-on edge $t=\tau_{0}$ where the precursor is in phase with the delayed main field. For comparison, we show the stacked optical transient responses from both two-level and EIT systems in Fig. 4. As shown in Fig. 4(a), a peak transmission larger than unity $(300 \%)$ is possible in the two-level system. In the EIT case, the constructive interference between the transient (300\%) and delayed main field (93\%) 


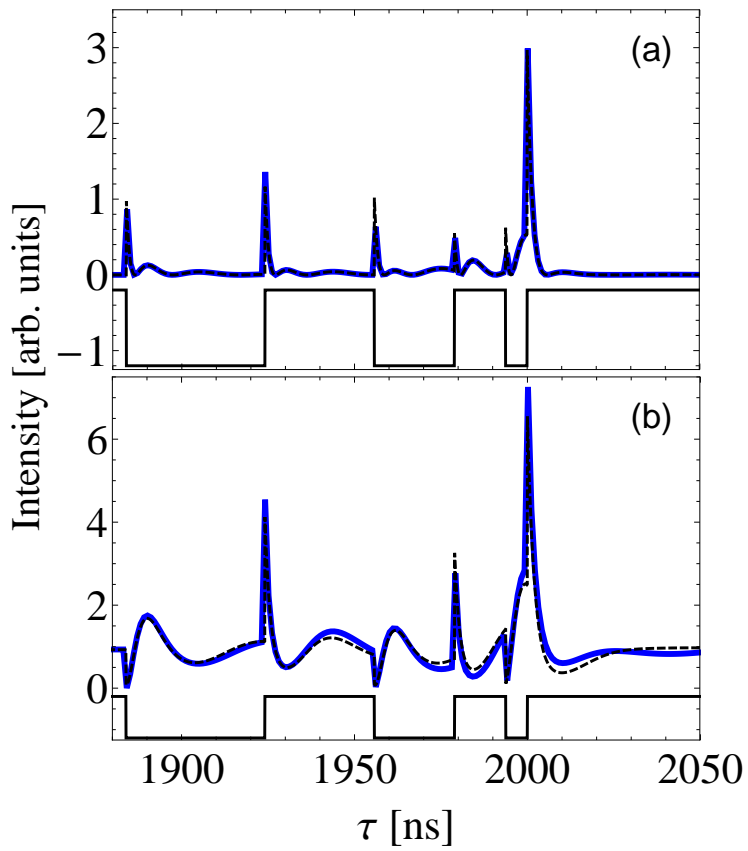

Fig. 4. (Color online) Normalized total intensities composed of stack of optical precursors and delayed main signal for the case of (a) two-level $\left(\Omega_{c}=0\right)$ and (b) EIT medium $\left(\Omega_{c}=4.2 \delta\right)$. The series of square pulses as input $E(0, t)$ is given as black solid line (shifted by -1.2). The hybrid analysis (Eqs. (4)-(6)), blue solid lines) is compared with FFT (black dashed lines).

leads to a $700 \%$ intensity peak. The blue solid lines, obtained using the hybrid-asymptotic method [Eq. (4)-(6)], display nearly identical features as that from FFT, except slightly different peak values resulting from the approximation of the hybrid analysis. Therefore, we identify that the stacked transients [4] are indeed an interference of optical precursors from different rising and falling edges. The black dashed lines are obtained using FFT with a time resolution of $50 \mathrm{ps}$. Due to the nature of FFT process, the 50 ps time resolution simulates a finite rise and fall time of $50 \mathrm{ps}$, and thus a bandwidth of 20 $\mathrm{GHz}$ that is commercially available. For the parameters given in this Letter, the optical transients generated from the square pulses with $50 \mathrm{ps}$ rise (fall) time haven been very close to that from ideal square pulses because there is no significant difference as we reduce the rise time from 50 ps to 5 ps. In a real situation, pulse generator and electronics also give rise to finite bandwidth. Thus, the theoretical prediction should be associated with the "low-pass filter" as used in Ref [5].

Discussion - We have studied optical precursors generated from step-on, step-off and square pulses in an EIT system. The long-lived oscillatory tails over $100 \mathrm{~ns}$ time at the falling edge results from the interference between the precursor and main field. We further extend the single square input pulse to a series of square pulses, and obtain about $700 \%$ peak transmission in the stacked optical transients. The agreement between FFT and hybrid- asymptotic methods confirms that the stack of coherent transients introduced by Macke et al. [4] is identified as the stack of optical precursors occurring at the rising- or falling edges of square pulses.

The authors acknowledge the valuable suggestions from the reviewers.

\section{References}

1. L. Brillouin, Wave Propagation and Group Velocity (Academic Press, New York, 1960).

2. K.E. Oughstun and G.C. Sherman, Electromagnetic Pulse Propagation in Causal Dielectrics (SpringerVerlag, Berlin, 1994).

3. M.D. Crisp, "Propagation of small-area pulses of coherent light through a resonant medium", Phys. Rev. A, 1, 1604 (1970).

4. B. Segard, J. Zemmouri and B. Macke, "Generation of electromagnetic pulses by stacking of coherent transients," Europhys. Lett. 4, 47 (1987).

5. H. Jeong, A.M.C. Dawes and D.J. Gauthier, "Direct observation of optical precursors in a region of anomalous dispersion," Phys. Rev. Lett. 96, 143901(2006).

6. E. Varoquaux, G. A. Williams, O. Avenel, "Pulse propagation in a resonant medium: Application to sound waves in superfluid 3B," Phys. Rev. B 34, 7617 (1986).

7. H. Jeong, U. L. Österberg, "Coherently transients: Optical precursors and $0 \pi$ pulses," J. Opt. Soc. Am. B 25 B1-B5 (2008).

8. H. Jeong, U. L. Österberg, "Steady-state pulse component in ultrafast pulse propagation in an anomalously dispersive dielectric," Phys. Rev. A 77021803 (R) (2008).

9. F.J. Lynch, R.E. Holland, and M. Hamermesh, "Time dependence of resonantly filtered gamma rays from Fe ${ }^{57}$," Phys. Rev., 120, 513 (1960).

10. J. E. Rothenberg, D. Grischkowsky, and A. C. Balant, "Observation of the formation of the $0 \pi$ pulse," Phys. Rev. Lett., 53, 552 (1984).

11. S. Du, P. Kolchin, C. Belthangady, G.Y. Yin, and S.E. Harris, "Subnatural linewidth biphotons with controllable temporal length," Phys. Rev. Lett. 100, 183603 (2008).

12. S. Du, C. Belthangady, P. Kolchin, G.Y. Yin, and S.E. Harris, "Observation of optical precursors at the biphoton level," Opt. Lett. 33, 2149 (2008).

13. H. Jeong and S. Du, "Two-way transparency in lightmatter interaction: Optical precursors with electromagnetically induced transparency," Phys. Rev. A 79, 011802(R) (2009).

14. B. Macke and B. Segard, "Optical precursors in transparent media," Phys. Rev. A 80, 011803(R) (2009).

15. D. Wei, J. F. Chen, M. M. T. Loy, G. K. L. Wong, and S. Du, "Optical precursors with electromagnetically induced transparency in cold atoms," Phys. Rev. Lett. 103, 093602(2009).

16. B. Macke and B. Segard, "Pulse normalization in slowlight media," Phys. Rev. A 73, 043802 (2006).

17. W. R. LeFew, S. Venakides, and D. J. Gauthier, "Accurate description of optical precursors and their relation to weak-field coherent optical transients," Phys. Rev. A 79, 063842 (2009). 